\title{
Revisión entre iguales y escritura académica en la universidad: la perspectiva del estudiante
}

\author{
Peer review and academic writing at \\ university: the student's perspective
}

Alfonso Vargas Franco ${ }^{1}$

Resumen

Este artículo presenta los primeros resultados de una investigación que tuvo como objeto documentar los puntos de vista (representaciones, grado de conciencia, actitudes, opiniones) que tiene un grupo de estudiantes, desde una perspectiva émica (una perspectiva del informante, del estudiante, que también es autor del texto), sobre la escritura académica, sus roles de autor y lector y los procesos de revisión entre iguales, en una secuencia de aprendizaje de resúmenes, reseñas y artículos académicos, en un aula universitaria. El corpus está constituido por una entrevista concebida como grupo de discusión, grabada y transcrita siguiendo el modelo del análisis del discurso, pero el estudio más amplio (Vargas Franco, 2013) describe y analiza entrevistas en profundidad y los textos académicos objeto de la revisión entre pares. El estudio de corte cualitativo-etnográfico arroja como resultados que la revisión entre iguales es más compleja de lo que parece, pero que puede resultar beneficiosa para que los estudiantes se apropien de las convenciones dominantes de la escritura académica, facilitando su proceso de integración en una nueva comunidad discursiva y cultural.

\section{Palabras clave}

Revisión entre iguales, escritura académica, representaciones, grado de conciencia, actitudes, perspectiva del estudiante.

\section{Abstract}

In this article we analyse the points of view (representations, awareness, attitudes, opinions) of a group of students from an emic perspective ('a perspective of the informant, of the student, who is also the author of the text'), regarding academic writing, their roles as author and reader and the processes of peer review, in a sequence of learning of abstracts, reviews and academic articles, in a university classroom. This text is based on an interview carried out as a discussion group, recorded and transcribed following the discourse analysis model. The qualitative-ethnographic study leads to results that the intersection of peers is more complex than it appears, but that it can be beneficial in that the students take charge of the dominant conventions of academic writing, facilitating their integration in a new discursive and cultural community.

\section{Keywords}

Peer review, academic writing, representations, levels of conciousness, attitudes, student's perspective.

Artículo recibido el 9 de noviembre de 2013 y aprobado el 14 de febrero de 2014

1 Universidad del Valle, Cali, Colombia. alfonso.vargas@correounivalle.edu.co 
A pesar del reconocimiento de la importancia que tiene detectar y reparar los problemas de los textos escritos académicos, la revisión de la escritura no es objeto de enseñanza sistemática en ninguno de los niveles de la escolaridad en Colombia, de acuerdo con la literatura revisada. Resulta todavía más preocupante que no se enseñe a los estudiantes de la universidad la revisión de sus propios textos en una institución que paradójicamente legitima la revisión de pares como requisito para validar la producción investigativa escrita (artículos científicos, reseñas, estudios e investigaciones). En efecto, la revisión entre pares legitima el campo de la escritura académica en la universidad y debería, por tanto, ser objeto de enseñanza sistemática en las instituciones de educación superior. Por otra parte, tampoco se ha explorado suficientemente la perspectiva del estudiante sobre la escritura académica y la revisión entre pares.

El sistema de revisión entre pares ha sido determinante para la producción de la disciplinariedad, al reforzar las ortodoxias particulares existentes en las comunidades académicas, los valores, normas y compromisos, permitiendo, de este modo, la estabilización de las prácticas de producción y circulación del conocimiento altamente especializado (StarkeMeyerring, 2010).

En el caso de la secuencia de aprendizaje de la redacción académica, objeto de indagación de este estudio, se implementó la revisión entre pares o revisión entre iguales como una estrategia para mejorar la composición escrita, pero también como una práctica para facilitar la inclusión de estudiantes que provienen de sectores populares - como los que ingresan en un alto porcentaje a la Universidad del Valle-, en una nueva comunidad de discurso. En otros trabajos (Vargas Franco, 2005, 2008, 2010, 2013) se expone la génesis de estas aproximaciones a la didáctica de la escritura en la educación superior y el papel que juega en ella la revisión entre pares, privilegiando la óptica del estudiante sobre este proceso.

Diversas investigaciones han corroborado la complejidad del proceso de apropiación de la lite- racidad académica, sobre todo en determinados contextos sociales en los cuales los estudiantes no han recibido una enseñanza específica en relación con los géneros discursivos propios de la universidad. Estos estudios concluyen, por una parte, que es necesario llevar a cabo una caracterización retórica y discursiva de estos géneros porque pueden aportar evidencia empírica para su enseñanza formal en el ámbito universitario, así como discutir una serie de tópicos que son el resultado de concepciones lingüísticas y psicológicas dominantes y que ofrecen una visión reduccionista de lo letrado (Cassany y Morales, 2009); y por otra, que existe un alto grado de aculturación que implica la literacidad académica (Castelló, 2009), que constituye el medio por el cual se accede a prácticas sociales de lectura y escritura que resultan prestigiosas y dominantes por el poder que otorgan a los sujetos y a las comunidades en que se insertan.

El concepto de aculturación que exponemos en este estudio se refiere a que ingresar a las comunidades académicas exige a los nuevos estudiantes apropiarse de una literacidad de tipo académico en las instituciones de educación superior para la cual generalmente no están preparados, sobre todo personas provenientes de contextos indígenas, afrodescendientes, campesinos y otros grupos minoritarios, así como estudiantes de zonas urbanas marginales. Esta situación puede producir conflictos de identidad en los alumnos indígenas, como la han documentado Zavala y Córdova (2010) en su estudio sobre la literacidad académica con estudiantes quechuahablantes en comunidades andinas del Perú.

Esta aculturación es producto del creciente ingreso a la universidad de grupos de personas cuyo acceso había estado restringido por razones económicas y sociales, tal fenómeno de masificación de la educación superior en los países de América Latina y la periferia ha supuesto una transformación de un modelo de educación superior para las élites para dar lugar a una universidad más abierta y plural.

De los planteamientos anteriores se revela como necesaria una aproximación más sociocultural, dado que se requiere rediseñar o ajustar la pedagogía de la 
lectura y la escritura en la universidad a las demandas, conocimientos y experiencias con las que cuentan los estudiantes que ingresan a esta nueva comunidad discursiva, provenientes en muchos casos de sectores sociales medios y bajos, para mencionar el caso de los sujetos de este estudio y que por distintas razones no fueron, salvo excepciones, aculturados en lo que Gee (1996) distinguió como un modelo de alfabetización basado en los valores del estilo de prosa de ensayo o "alfabetización ensayista" (Scollon R. y Scollon S., 1981; en Gee, 1996). Este modelo del discurso de la alfabetización ensayista o essayist literacy training ha constituido el eje de la formación de la cultura dominante en Estados Unidos, Canadá y en general en el mundo anglosajón.

El rediseño o ajuste de la pedagogía de la lectura y la escritura en la universidad implica reconocer, por un lado, que la literacidad académica no es neutral y transparente (Zavala, 2009, 2011), y por el otro, que la enseñanza de los géneros discursivos, y de los procesos de composición de textos académicos, debería ir encaminada no solo a una aculturación en una nueva comunidad discursiva, sino también a que los recién llegados puedan beneficiarse de los poderes que brinda el dominio de la literacidad académica.

Sin embargo, el estudio que presentamos aquí se fundamentó inicialmente en el marco constructivista sobre la enseñanza de la escritura (Flower, 1994; Wells, 2001; Cassany, 1999; Camps, 1994) y los enfoques sobre literacidades académicas (Lillis, 1997; 2001; 2003; Ivanic, 1998; Zavala, 2009, 2011; Zavala y Córdova, 2010). Por lo tanto, es preciso aclarar que el desarrollo de la investigación comenzó bajo un enfoque cognitivo representado en el marco constructivista de la enseñanza de la escritura académica porque tuvo como eje la revisión entre pares que es una herramienta propia de las corrientes epistemológicas del aprendizaje colaborativo y cuyo sustento teórico se encuentra en los trabajos de Vygostky; pero luego, al documentar los puntos de vista de los sujetos (sus representaciones, el grado de conciencia, las actitudes y las opiniones) que tiene un grupo de estudiantes, desde una pers- pectiva émica (una perspectiva del informante, del estudiante, que también es autor del texto), sobre la escritura académica, sus roles de autor y lector y los procesos de revisión entre iguales, en una secuencia de aprendizaje de resúmenes, reseñas y artículos académicos, en un aula universitaria, constatamos que se requería una mirada más sociocultural sobre la perspectiva del estudiante, asunto que no se había explorado hasta el momento de manera profunda con el marco constructivista de la enseñanza de la escritura descrito arriba.

La perspectiva de las literacidades académicas nos permitía una mirada más rica y amplia sobre la perspectiva de los sujetos en torno, por un lado, a la revisión entre iguales y, por el otro, a la escritura académica como práctica que adquiere un significado cuando el sujeto es capaz de objetivarla, pero al mismo tiempo de articularla con sus experiencias y saberes previos, sus motivaciones, sus deseos y sus aprensiones en torno a ella.

En este marco contextual y teórico, nos propusimos implementar en un curso de composición escrita (Composición en Español I) la enseñanza de la práctica de revisión entre iguales o revisión entre pares. Preferimos el nombre de revisión entre iguales porque connota un significado más democrático y se diferencia de la revisión entre pares que se emplea en las revistas científicas para los procesos de arbitraje de los artículos académicos. Sin embargo, reconocemos la complementariedad de propósitos y dinámicas de ambos tipos de prácticas.

La revisión entre iguales puede definirse como una práctica, dispositivo o actividad didáctica que consiste en planificar la producción escrita en una secuencia de aprendizaje que requiere la escritura de borradores, los cuales son intercambiados, leídos y comentados por un compañero antes de la revisión final del profesor.

El subproceso de revisión es considerado por muchos estudiosos como el más importante dentro del proceso global de la composición (Camps, 1994). En contraste, se trata de uno de los campos menos estudiados y desconocidos. Además de constituirse en un aspecto que revela la diferencia entre escri- 
Universidad Pedagógica Nacional

Facultad de Humanidades

tores expertos y aprendices (Cassany, 1993, 1999). Es por esta razón que exploraremos la interacción entre iguales, mediada por el profesor, que se desarrolló en un curso de composición en español que hace parte del programa de Licenciatura en Lenguas Extranjeras en la Universidad del Valle (Colombia), cuyo eje didáctico fue la revisión entre iguales de los textos de los estudiantes.

\section{Investigaciones previas}

Si bien la revisión entre iguales con guía del docente es una práctica habitual en los programas de "escribir a través del currículo" (Writing across the curriculum), propios de las corrientes anglosajonas que integran la enseñanza de la escritura académica en las diferentes disciplinas, lo es mucho menos en tradiciones escolares no anglosajonas, España y los países de América Latina, por ejemplo, y Colombia específicamente. Sin embargo, existe en el contexto hispanoamericano un reciente conjunto de investigaciones que han corroborado las ventajas de la revisión colaborativa o revisión entre iguales (Carlino, 2004, 2008; Castelló, 2009, 2008; Castelló, González e Iñesta, 2008) en la escritura de tesis de posgrado y en trabajos de investigación con alumnos de grado, así como trabajos predecesores que apoyados en enfoques discursivos derivados de la tesis de la concepción sociocultural del lenguaje y la significación de Vygostky, defendieron la importancia de la interacción, de la colaboración y de los intercambios verbales entre iguales durante el proceso de composición escrita, (Cassany, 1999), así como la importancia de la revisión y la necesidad de enseñar a revisar a maestros y estudiantes (Cassany, 1993).

Desde la perspectiva de la investigación-acción, Carlino (2004) analiza las propuestas pedagógicas que se pusieron en práctica en dos talleres de escritura de dos maestrías. Las preguntas que examinó en este ciclo de investigación-acción fueron cómo prevenir el aislamiento y la discontinuidad que experimentan los tesistas cuando finalizan sus ciclos académicos y deben enfrentar la escritura de la tesis; cómo brindar apoyo a los tesistas para redactar sus trabajos de fin de máster, si se tiene en cuenta que no han tenido tiempo en la clase presencial para discutir sus borradores; y finalmente, qué dispositivos pueden emplearse en los talleres de tesis para que los estudiantes expresen su experiencia vital. Las actividades realizadas fueron la elaboración de un diario de tesis y la revisión entre pares. Uno de los problemas que detectó Carlino, al final del ciclo investigativo, fue haber dejado la revisión entre iguales a la autogestión de los maestrandos y por fuera del aula. Esto llevó a la investigadora a incluirla dentro de los encuentros presenciales organizados para comentar los trabajos escritos de cada estudiante con ayuda de un guía. Ahí, la valoración de los sujetos fue positiva. Es decir, que para que la propuesta de la revisión entre pares funcione ha de ser incluida en el currículo y desarrollada dentro de los espacios presenciales del taller de escritura de tesis con la ayuda del profesor.

En definitiva, se hace necesario superar la brecha entre las intenciones del docente y el punto de vista de los estudiantes. Para estos no es suficiente conocer los potenciales beneficios de llevar a cabo la propuesta, de poder hacerlo, sino que por el contrario, necesitan que la práctica esté andamiada externamente. En otras palabras, requieren que el tutor lleve a cabo una labor de mediación más profunda y extensa para comprender las ventajas de la revisión entre iguales.

En un estudio posterior, Carlino (2008) parte del contexto de la baja tasa de graduación que se presenta en los posgrados argentinos, concretamente en las maestrías de las ciencias sociales. Las dificultades de los estudiantes para concluir sus tesis constituyen ya un objeto de estudio de numerosas investigaciones internacionales, así como la dificultad que presentan para revisar sus escritos. La enseñanza de la revisión entre pares (Dysthe, Samara y Westheim, 2006, en Carlino, 2008) se enmarca en la situación de los posgrados descrita. En el estudio se examina de nuevo como eje didáctico la revisión entre iguales dentro de una serie de talleres de escritura de proyectos y avances de tesis en diferentes posgrados. Los objetivos de la actividad de la revisión entre pares son: 
1. Promover que los tesistas reciban comentarios de potenciales lectores que les ayuden a detectar y solucionar problemas de sus textos escritos.

2. Que los alumnos se enfrenten a una "audiencia de prueba".

3. Contribuir a la explicitación de criterios para que los estudiantes revisen sus propios escritos.

4. Propiciar el intercambio entre pares para paliar la falta de un apoyo continuo en su labor como tesistas.

En el estudio se defienden varias razones para incluir la enseñanza de la revisión entre pares en el currículo del taller de escritura de posgrado, que compartimos en nuestro trabajo en pregrado. En primer lugar, la revisión entre iguales debe ser enseñada porque es una práctica social que caracteriza la comunidad científica. En segundo lugar, la revisión entre pares debe ser objeto de la didáctica de la escritura en los diferentes niveles de escolaridad y especialmente en la universidad porque se han corroborado por parte de profesores e investigadores (Boote y Beile, 2005, en Carlino, 2008) los diferentes problemas que tienen los alumnos para escribir. En tercer lugar, la revisión entre pares en los posgrados indica que revisar el texto de un compañero $y$, a su vez, recibir sus comentarios sobre el trabajo propio sitúan al estudiante en un rol más activo y más fértil para aprender. En cuarto lugar están esta serie de razones: 1) Es más fácil ver problemas en el texto ajeno que en el propio; 2) la revisión entre pares subdivide el proceso de escritura en diferentes fases, lo cual puede incidir en disminuir la carga cognitiva y; 3) finalmente, enseñar la revisión entre pares revela que los académicos, comunidad a la que aspiran pertenecer los tesistas ha de saber revisar a fondo y de manera frecuente textos ajenos y propios. Por otra parte, como en otros trabajos (Carlino, 2005), se insiste en la necesidad de que a nivel institucional se reflexione sobre el valor de la escritura y se le asigne un lugar en el currículo a su enseñanza. En este orden de ideas, se pide institucionalizar la enseñanza de la revisión entre pares a través de encuentros periódicos entre revisores en espacios como los "círculos de escritura de tesis" (Airchison, 2003; en Carlino, 2008).

Los resultados de los sucesivos ciclos de investigación-acción referenciados en este estudio permiten delimitar tres grandes aspectos:

1. Problemas para llevar a cabo la revisión entre pares.

2. Condiciones para que funcione la tarea.

3. Aspectos que los estudiantes valoran como positivos.

Los principales problemas tienen que ver con que los tesistas inician equiparando "revisión" con "evaluación sancionadora"; no saben qué comentar del trabajo de sus compañeros y cuando lo hacen pueden ser demoledores. En conclusión, sus comentarios iniciales son de baja calidad y poco útiles. Desconfían, además, de aportar algo valioso y de recibir, a su vez, comentarios a sus textos que les sirvan; prefieren, en cambio, las correcciones del profesor.

Por otra parte, si no se logra un trabajo sostenido durante la clase dedicado a la revisión colectiva y entre pares, los estudiantes no saben que la lectura de un igual puede aportarles puntos de vista $\mathrm{e}$ información sobre el efecto retórico del texto en los lectores. La inexperiencia en la revisión entre pares impide el reconocimiento de su potencial utilidad para mejorar el texto propio y crecer como escritores. Por lo tanto, es importante enseñarla ofreciendo reiteradas oportunidades para ponerla en práctica.

En cuanto a las condiciones para que funcione la tarea resulta necesario diseñar una serie de condiciones didácticas para favorecer su implementación. Por un lado, heterorregularla (orientarla y retroalimentarla) y, por el otro, dedicarle tiempo en clase para que se viva la experiencia de su utilidad. Ofrecer, por ejemplo, modelos explícitos e implícitos sobre cómo llevar a cabo la práctica, qué aspectos comentar de los textos, evitando comentarios agresivos y motivando a que los productores encuentren que su texto puede responder mejor a las intenciones comunicativas del autor. 
Universidad Pedagógica Nacional

Facultad de Humanidades

Impulsar, así mismo, a que la tarea se lleve a cabo fuera del tiempo presencial del taller proveyendo a los estudiantes de pautas precisas, preguntas "tipo" para formular al texto del par, etc. Otra condición tiene que ver con la formación de los grupos de pares: ¿Quiénes conforman las parejas de revisores mutuos?, ¿cómo han de ser los revisores para que se sientan autorizados a ofrecer su lectura crítica y acepten el comentario del par?, ¿es viable que dos parejas, a su vez, interactúen entre sí, de tal manera que cada estudiante-autor reciba varias revisiones de distintos lectores?

En cuanto a los aspectos valorados como positivos por los alumnos, la investigación concluye que, en primer lugar, reconocen la revisión entre iguales como una estructura de apoyo, un "ámbito social de pertenencia" ausente en la mayoría de posgrados; en segundo lugar, los alumnos reconocen que es una herramienta que ayuda a aprender criterios para reconsiderar los escritos propios, y que anticipar la revisión del par lleva a la autorregulación. Así mismo, la revisión entre pares contribuye a tener en cuenta el punto de vista del lector. Finalmente, la revisión entre pares permite tener un lector auténtico. Los tesistas también reconocen el valor epistémico de la actividad y también recomiendan ampliar la tarea e iniciarla desde el ciclo de pregrado. Como aspectos más conflictivos, el estudio detectó que los maestrandos cuestionaron el hecho de que no se consiguió dejar una estructura armada para que los alumnos hicieran uso de los grupos de revisores al finalizar los seminarios.

Para finalizar, la investigación concluye que dar un espacio a la enseñanza de la revisión entre pares, a corto o mediano plazo, se justifica porque quienes deseen hacer parte de la comunidad científica o profesional lo lograrán de manera más eficaz si antes se les permite escuchar de parte de algún colega los comentarios a sus borradores y si aprenden a revisarlas desde la perspectiva de sus potenciales lectores.

En una propuesta de intervención dentro de un proyecto de mejora de la formación docente que se desarrolla en los estudios de psicología (Castelló,
2009), se implementaron varias estrategias didácticas integradas y coordinadas para mejorar la escritura de trabajos de investigación de los estudiantes como su proyecto final de grado. Dentro de las estrategias didácticas interesa, para los fines de nuestro estudio, el dialogar con otros autores. La finalidad de esta estrategia es conformar una comunidad de escritores que asumen la responsabilidad de sus decisiones y son capaces de dialogar con otros desde su posición de autor. Se formalizó el espacio con el nombre de Seminario de Trabajo de Investigación, un espacio para que los estudiantes presentaran sus avances y comentaran sus dificultades en el proceso de escritura. Antes de concluir el texto, esto es, su versión final, los alumnos revisan de manera pormenorizada el trabajo de uno de sus compañeros - su pareja de escritura-, y proponen formas de mejorarlo, con el acompañamiento de una guía de revisión. El estudio concluye que en la discusión conjunta aparecen, se definen y se proponen las soluciones. El hecho de que los alumnos dispongan de la guía, hayan compartido las sesiones de seminario, genera entre sus integrantes la idea de que no están solos en este proceso, que tiene efectos positivos en el plano afectivo y les brinda la posibilidad real de que disponen de un conocimiento compartido y un lenguaje común frente al proceso de composición; un hecho indispensable para que las parejas de revisores funcionen de forma verdaderamente colaborativa.

\section{Metodología y corpus}

\section{Objetivos y preguntas de investigación}

El objetivo general de este estudio es describir y analizar las concepciones, actitudes, opiniones, representaciones, grado de conciencia, etc., que tiene un grupo de estudiantes, desde una perspectiva émica, sobre la enseñanza de la práctica de revisión entre iguales en el aula y su incidencia en el proceso de construcción de la escritura académica, en sus roles de lector $y$ autor $y$ su posible incidencia en su formación como docentes, en una secuencia de aprendizaje de resúmenes, reseñas y artículos académicos, en un aula universitaria. 
Las preguntas de investigación se concretan de la siguiente forma:

1. ¿Cuáles son las opiniones, actitudes y creencias de los estudiantes sobre los logros y las dificultades que experimentan en relación con la práctica de la revisión entre iguales?

2. ¿Qué creencias y saberes construyen los estudiantes sobre la escritura académica y sus géneros a partir de la enseñanza de la revisión entre iguales?

3. ¿Qué grado de conciencia alcanzan los estudiantes sobre su propio proceso como escritores de textos académicos a partir de la implementación de la práctica de revisión entre iguales?

4. ¿Se producen algunos cambios en las representaciones de los estudiantes sobre la revisión en particular y sobre la escritura académica en general después de la implementación de la práctica de revisión entre iguales?

5. ¿Qué aspectos afectivos y emocionales emergen durante la práctica de la revisión entre iguales? ¿Cómo los enfrentan los estudiantes?

6. ¿Qué cambios se experimentan en las representaciones sobre el rol del docente durante el proceso de implementación de la práctica de revisión entre iguales?

7. ¿Cuáles son las representaciones que los estudiantes construyen sobre la incidencia de la revisión entre iguales en su futuro como docentes de lenguas?

Este estudio hace parte de una investigación de mayor envergadura concebida como un estudio de caso, con trazas de investigación-acción y etnografía que está orientada a analizar, por un lado, las ventajas de implementación de un enfoque constructivista sobre el lenguaje escrito en la universidad y, por el otro, apunta a producir algunas transformaciones curriculares y didácticas en la enseñanza de la escritura en la universidad - generalmente hegemónicas y monológicas- para propender por prácticas más democráticas e incluyentes, a partir de un enfoque sobre la perspectiva del estudiante.

El corpus está conformado por la transcripción de un grupo de discusión, específicamente por las voces (testimonios) de los sujetos que participaron en el diseño e implementación de una secuencia didáctica y evalúan sus concepciones y representaciones sobre la escritura académica y el proceso de composición de textos académicos, a partir de la implementación de la práctica de revisión entre iguales mediada por el docente.

El grupo de discusión se grabó en audio el día 18 de febrero de 2010, casi un año y medio después de finalizar la secuencia de aprendizaje, centrada en la revisión entre iguales, en el mes de julio de 2008. Participaron 11 estudiantes cuyas edades oscilan entre los 20 y 34 años, y tuvo una duración de 1 hora y 10 minutos (33 páginas de transcripción). Los estudiantes pertenecen a estratos sociales medios y bajos. La entrevista tuvo lugar en el salón del Grupo de Trabajo de Idiomas (GTI), contiguo al Edificio de la Escuela de Ciencias del Lenguaje, a la cafetería de Idiomas y a la biblioteca Mario Carvajal de la universidad. Es un aula asignada a los estudiantes para que realicen actividades extracurriculares o extraacadémicas (preparación de reuniones, organización de eventos culturales y políticos, etc.). Sin embargo, el salón ha ido ganando con el tiempo, un valor simbólico agregado y es el de la contestación política porque ha sido empleado por grupos de estudiantes que participan en tareas de contestación frente al establecimiento social en Colombia. Lo escogimos por razones prácticas: una mesa rectangular, con largos asientos, y cierta privacidad. Los salones de clases del edificio de la escuela estaban cerrados porque no había iniciado formalmente el período académico.

La transcripción se realizó siguiendo los parámetros del análisis del discurso en general y del análisis de la conversación en particular (Duranti, 1997, 2000; Brown y Yule, 1983, 1993; Payrató, 2003; Tusón, 1997). Se hizo una transcripción ortográfica que incluyó aspectos de la transcripción fonética como solapamientos, pausas, alargamientos vocá- 
Universidad Pedagógica Nacional

Facultad de Humanidades

licos y otros aspectos prosódicos (pronunciaciones enfáticas, débiles, risas), así como aspectos de la comunicación no-verbal o gestual. No obstante, en la transcripción que presentamos en este texto no nos proponemos un análisis del discurso oral de los sujetos, sino recuperar sus voces para apuntalar la perspectiva del estudiante sobre la revisión entre iguales.

\section{Resultados y discusión}

Considerando la perspectiva de los estudiantes, algunos de los resultados más significativos relativos a sus concepciones, representaciones y opiniones sobre la escritura académica y concretamente sobre la práctica de la revisión entre iguales son los siguientes:

\section{Recuperando la memoria}

En este apartado se describen y analizan las valoraciones sobre la revisión entre iguales, el grado de dificultad que tiene la práctica, las ventajas que le atribuyen los estudiantes, los ajustes que deberían hacerse dentro del dispositivo y, finalmente, el significado personal que atribuyen a la práctica.

Es de anotar que durante los primeros minutos del grupo de discusión, hubo que reconstruir la memoria de la secuencia de aprendizaje, como una manera de "romper el hielo" y de recuperar "el tiempo perdido". Entrevistador e informantes volvían a encontrarse 17 meses después. Los estudiantes estaban un poco sorprendidos, según lo revelaban sus gestos. Este hecho representó una ventaja para la validez de los resultados del estudio por cuanto que se había producido un distanciamiento entre profesor y estudiantes que podría contribuir a una mayor objetividad y desapasionamiento por parte de los informantes. El entrevistador preguntó inicialmente la opinión de los estudiantes sobre el papel del trabajo sobre escritura académica y revisión entre iguales en su vida personal y académica y, una de las informantes planteó: "Dora: Debíamos hacer como un recorderis, ¿no?”. A partir de esta pregunta-petición se desencadenó todo el recuerdo y posteriormente la discusión. Varios estudiantes
Jimena, Adela, Andrea ${ }^{2}$ y el profesor empezaron a encadenar los hitos del proceso.

El entrevistador preguntó a los estudiantes sobre el significado que atribuían en su vida personal y académica al trabajo sobre escritura académica. $\mathrm{Al}$ respecto una de las estudiantes más comprometidas con el proceso de revisión entre iguales expuso su punto de vista:

Andrea: Yo pienso en la vida personal es importante porque porque nos ha ayudado como a aceptar el el criterio del compañero; es decir, yo sé que para algunos puede ser incómodo que que una persona que no es el profesor le lea el trabajo y como que le diga, pues como que trate de corregirlo, entonces esa parte, yo pienso que que en la vida personal e importante para para aprender a aceptar el criterio del compañero, para para aprender a ser criticado.

La informante reproduce en su discurso la opinión de varios compañeros que participaron en la práctica. En definitiva, a lo largo de la discusión tomó distancia de esta postura y defendió la importancia de la revisión de un igual. Del mismo modo otros estudiantes valoran el mayor grado de autonomía al que puede conducir la práctica de la revisión entre iguales en el aula y la cesión de la responsabilidad del profesor hacia los mismos alumnos.

Otro aspecto que destacan los estudiantes es que cada uno tiene su estilo, pero que puede tomar ideas de los otros, discutir sobre el propio proceso de escritura y que la escritura es un proceso de revisión.

Adela: Que cada cual tiene su forma diferente de expresarse [silencio], pues creo que es eso y que uno puede tomar ideas de los otros, umm, complementarlas y discutir entre iguales qué estoy haciendo bien y de pronto qué no estoy haciendo bien.

Jimena: Pues algo que me quedó a mí en este proceso, es precisamente que la escritura no es algo que uno haga en un día y ya!, escribiste el texto y así quedó, sino que es un proceso de revisión, un proceso en el que uno tiene que ir mejorando ese

2 Nombres ficticios de los informantes del estudio. 
texto, de pronto por eso en Composición II [el curso siguiente que fue impartido por otro profesor y con otra propuesta didáctica] me rehusaba un poco cuando nos tocó hacer el primer taller evaluativo donde había que producir un texto en dos horas [...], igual me queda que debe ser un proceso y no no no algo que uno haga en un día, en una hora, en un momentico que me quedó libre.

Jorge: Pues a mí me quedó que digamos, a mí me quedó como la humildad y la modestia de comprender que el otro también es es una ayuda, una ayuda bastante importante; de que uno no debe negarse a solicitar la colaboración de otros, si es necesario.

\section{Conciencia sobre el propio proceso de escritura}

En este apartado se exploran los conocimientos sobre la estructura de los textos y sus niveles de organización semántica (coherencia y cohesión), así como sobre la complejidad de la escritura académica, sobre la importancia de la revisión, sobre sus avances.

La mayoría de los participantes en el grupo de discusión reconocen que la experiencia del curso Composición en Español I y el trabajo desplegado en la secuencia de aprendizaje en torno a la revisión entre iguales, cambió muchas de sus concepciones y prácticas sobre la escritura académica en general y sobre la revisión en particular. Es decir, a pesar de la incomodidad inicial y la resistencia a ser revisados por un igual, todo el proceso desarrollado en el aula, incluida la revisión entre iguales, es valorado positivamente por los estudiantes que coinciden en destacar que hay un cambio muy grande en la forma como escriben. Comparan el antes y el ahora en su dimensión como escritores y constatan que el conocimiento sobre la estructura de los textos y la revisión les ayudaron a mejorar sus habilidades como escritores. Antes se sentían bloqueados por la dimensión de la tarea:

Andrea: Yo estuve ayer buscando mis trabajos de de de Composición, del curso de Composición II y con los otros cursos como como todo el proceso que hemos llevado, y uno se da cuenta de que realmente hay un cambio grandísimo en

el en el la forma como uno escribe, en la forma como redacta uno; en ese tiempo para mí, por ejemplo, era muy difícil escribir; es decir, yo me podía quedar estancada dos horas sin sin poder pasar de una línea porque porque no sabía cómo desarrollar el texto.

Dentro de los saberes que los estudiantes afirman haber construido durante el proceso, cabe destacar el reconocimiento de la audiencia o el público para el cual se escribe, así como el proceso que implica la composición de un texto escrito de carácter académico. Inicialmente creían que escribían para el profesor. Cuando el entrevistador les preguntó: “¿para qué tipo de público escribían ustedes?” Respondieron en voz baja y casi al unísono: "para el profesor".

Además, del reconocimiento de la audiencia, el procedimiento o el cómo y los conceptos, estas escrituras académicas se producen para ser publicadas, lo que implica reconocer su carácter social.

Jorge: Lo primero que yo aprendí en clase, sinceramente eso fue lo primero que yo aprendí, ese tipo de conceptos que uno no tenía claros ni definidos sobre a quién se escribía, cómo se debía escribir [...] pero también aprendí que también hay que escribir para un público más general, ya en ese ambiente uno ya no escribe para los profesores, sino que esas escrituras pueden postularse para ser publicadas, es muy importante que lo que uno escriba pueda ser entendido por todos.

Moisés: Lo importante para mí fue eso, darme cuenta que yo podía, eh cuestionarme sobre la forma en que escribía porque uno realmente no escribe para uno mismo, para alguien que lo va a leer a uno.

Sin embargo, el desconocimiento sobre las características de los géneros académicos también constituyó para algunos estudiantes un problema durante el desarrollo de la práctica de revisión entre iguales:

Claudia: No, pues, siempre fue como me sentía débil, pues para analizar el texto del otro compañero, si ni siquiera el mío, pues ni siquiera sabía si estaba bien, eh, además porque si había, tenía uno como 
Universidad Pedagógica Nacional

Facultad de Humanidades

que entrar más, si como analizar las estructuras o y si uno no conocía bien la estructura de ciertos textos, entonces era difícil también como juzgar el del otro, ¿si me entiende?

El caso de esta estudiante llama la atención porque fue una de las personas que más revisó y con mejor criterio; además el profesor a lo largo del curso explicó la estructura de los textos trabajados en clase a partir de los aportes de la lingüística del texto y el análisis del discurso.

La conciencia sobre los propios errores cobra fuerza en algunos estudiantes como parte de su propio de reflexión metacognitiva.

Gustavo: Es que yo soy una persona que tengo todas las ideas en la cabeza, pero al momento de argumentar, yo me riego y yo hago, yo soy malo pa' escribir, entonces tengo una idea muy fundamentadas, pero al escribir la hago mal, la hago errónea [...] la idea que yo tenía la argumenté mal, la estructuré mal, en organización de las ideas; yo escribo y yo creo que está bien, eso, como que creo una mezcla de todas las ideas que tengo y no las estructuro bien.

Esteban: En la escritura pasé de ser un loco más, a ser un poco más, más, más consciente pero más ordenado, es la vaina porque a uno de le venían las ideas a la cabeza, y uno prummm, empezaba a llenar un párrafo y hacía un párrafo de 20 líneas, con, hasta sin puntos y sin nada, entonces uno escribía a la loca; lo que me quedó fue aprender a estructurar, a estructurar mis textos.

De los géneros producidos a lo largo del curso, el que produjo mayor dificultad a los estudiantes fue el ensayo. Aquí nos referimos al artículo científico, por contraposición al ensayo especulativo o literario, heredero de la línea de Montaigne. Al final de la secuencia de aprendizaje, los alumnos debían escribir un ensayo académico que incluía resumen y abstract, introducción (propósito, perspectiva teórica, importancia de la cuestión), desarrollo y conclusiones. Cuando el entrevistador preguntó: “¿Cuál fue el trabajo que de pronto les generó más dificultad?", los estudiantes respondieron: “el ensayo”, casi al unísono. Los informantes atribuyen este problema al desconocimiento del género, por un lado, y a la escasa o nula enseñanza recibida en la educación secundaria. El entrevistador preguntó luego: “¿Por qué?, ¿por la complejidad del mismo?” Y una de las estudiantes respondió:

Adela: ¡Porque nunca había escrito uno!

Entrevistador: nunca habían escrito uno.

Adela: En el colegio en el colegio a mí nunca me pusieron a hacer un ensayo; yo vine a escuchar la palabra ensayo en la universidad [...] seguro.

Gustavo: Uno creía que, como un resumen, era como la estructura de un resumen.

Jorge: Es que en el colegio, en el colegio a uno lo ponían a escribir ensayos y uno pensaba que simplemente era coger dos ideas y contrastarlas y iya!, pero entonces aquí uno ya se viene a dar cuenta cuando uno realiza un ensayo, así, bien largo que un curso de casi 6 meses, uno se da cuenta de que es bastante complejo porque son varias, son analizar todas las ideas; primero se tiene que hacer la macroestructura, definir cuál es la hipótesis, bien cuál es el tema, definir cómo va a ser uno de los párrafos a las proposiciones y cada párrafo trabajarlo y después como un ente aparte y al final tener todo combinado, pues es entonces bastante complejo realizar un ensayo.

Finalmente, queda una mayor conciencia crítica sobre el propio proceso de escritura, de manera especial con respecto a la revisión de los textos propios y apertura al juicio de los otros.

Andrea: Siempre tengo presente, en mis textos, eso; yo pienso, es decir, uno muchas veces uno siempre que hace un texto, uno piensa que ahí, bueno, ya lo terminé, aquí está, se lo entrego, pero para mí siempre es importante como leerle [...]; siempre como pensar de una manera crítica hacia lo que estoy escribiendo, como siempre tratar de mejorarlo, si, si, es posible eso y también pues me pareció superenriquecedor, eh, la experiencia de tener a alguien que te corrija, que uno se pueda enfrentar a eso, ah, lo que decía al principio, a ser criticado, es [pausa] muy interesante. 
Jimena: Que la lectura y la escritura también es un proceso también, que el hecho de releer también le hace a uno entender cosas nuevas, es un proceso y que realmente uno puede volverse más crítico respecto al trabajo propio y al del otro

\section{Aspectos afectivo-motivacionales e identitarios}

En este ítem se exploran asuntos tales como la escogencia de la pareja de escritura, así como los sentimientos de inseguridad, desconfianza frente al propio saber sobre la escritura y sobrevaloración del papel del profesor. Así mismo, se evidenció una reflexión sobre el mayor grado de confianza con el compañero que con el profesor y finalmente las posturas opuestas o ambivalentes en este sentido.

Varios informantes (Gustavo, Dora y Andrea) mencionan que los temores frente a la revisión entre pares están fundamentados sobre la creencia de la propia falta de competencias o capacidades, y en las de sus compañeros para llevar a cabo un trabajo de revisión más riguroso. Sin embargo, frente a la pregunta del entrevistador sobre si la revisión individual o la revisión entre iguales pueden ser mejores o más productivas, consideran que la mirada de otra persona es muy importante para detectar problemas o errores en el texto propio.

Asociado a este aspecto se encuentra la sobrevaloración de la figura del profesor, a quien, salvo excepciones, se le considera la persona más idónea para hacer la revisión.

Gustavo: Profe, yo pienso que eh eh, el hecho de, el hecho de que un compañero a uno le, por decir le corrija el texto a uno, uno inconscientemente no lo acepta [...]; entonces uno nunca va a aceptar que una persona, por decir, igual que lo critique porque no está como a la altura de hacerlo...

Pedro: Yo necesito que el profesor me lo revise, porque él va a ver más cosas; él va a ir más allá y entonces me va a retroalimentar mejor.

Sin embargo, es un aspecto controversial porque otros estudiantes refutan este planteamiento, afirmando que les genera más confianza la revisión de un igual y que concepciones dominantes sobre la enseñanza han llevado a creer que "el profesor es el que tiene la palabra”. No obstante una de las estudiantes reivindica la necesidad de revisar los trabajos con una "perspectiva de estudiante" que esté, según se infiere de su respuesta, más cercana al proceso de escritura del alumno:

Dora: ¡Síl, hay una resistencia, y lo que decía Andrea, como que uno no acepta totalmente el criterio de otras personas que se supone son pues pares, si? Es más fácil que el profesor o alguien con más autoridad lo haga, pero por ejemplo a mí me pasa, me pasaba y me pasa que yo yo no tengo mucha seguridad en mis escritos, entonces era bueno, o sea era muy positivo en cuanto a tener como la opinión de alguien más, me ayudabaaa [...], o sea en cuanto a la seguridad con sus propios escritos era un punto muy positivo.

\section{Una perspectiva de estudiante}

Andrea: Es decir el profesor es el que tiene la palabra, entonces él es el que revisa, entonces es el que le puede decir si está bueno o está malo, pero para mí es importante la la opinión del compañero porque puede ser que a uno no se le ocurrió una idea y el compañero te diga, ah, puedes colocar esto aquí y tiene como una perspectiva, o sea una perspectiva de estudiante, una perspectiva de de del texto, de lo que estamos haciendo que es diferente a la que tiene el profesor, si me entiende?

Por otra parte, también influye la elección de la pareja de revisión. En todo caso, los estudiantes preferirían hacer la práctica con una persona con la que tuvieran mucha confianza, un amigo dentro del curso. Esta creencia se justifica por el temor a la crítica grosera o irrespetuosa de alguien con quien no tuviesen mucha cercanía, aunque tampoco hubo evidencias o quejas en el sentido de que hubiera sucedido durante la revisión. Los alumnos consideran que si tienen confianza con su compañero de revisión, esto los hacía sentir más libres de decir toda la verdad sobre el trabajo del otro. Una creencia más que se evidenció en la discusión fue considerar que el hecho de que cada estudiante tiene su estilo y que este no debería ser modificado o alterado. En definitiva, valoran la cortesía que debe haber en le 
Universidad Pedagógica Nacional

Facultad de Humanidades

revisión entre iguales y piensan que la confianza no implicaría menos rigor en la corrección:

Esteban: Pues yo creo que a veces uno se sentía cuando trabajaba, cuando le revisaba a otra personas que no tenían mucha confianza. En mi caso particular me sentía muy limitado, o sea muy limitado a decirle cambia todo, o sea era cambiarle de pronto una oración, que a mi parecer no estaba bien redactada, como cambiarle todo, me sentía muy limitado aaa decirle vé a...

Entrevistador: "Proponerle", interpela el profesor.

Esteban: Proponerle, ve cambiá todo [risas de varios chicos y chicas].

Entrevistador: ¿Y querías decirlo?

Esteban: Sí, cambiá todo, pero sí, por dentro queriéndolo decir, o sea le decía, o sea no es capaz de decirle tan...

\section{Entrevistador: Espontáneo.}

Esteban: Sí, tan directamente.

Para otro estudiante, Moisés, el problema no era tanto el grado de proximidad emocional con la persona, sino que no revisaba sus propios textos, que no tenía la experiencia necesaria:

Moisés: Cuando a mí me tocaba que me revisaran el texto, de pronto para mí el hecho de que fuera una persona cercana para mí o no, no me preocupaba mucho; a mí lo que me preocupaba era que a mí tampoco, o sea yo no revisaba mis textos; yo escribía y el texto como quedó, ahí...

Pero también hubo personas que temían la revisión del profesor y se sentían más tranquilos confiando la revisión a un igual. Este hecho puede estar ligado también a la sobrevaloración de la función docente:

Claudia: Profe, para mí fue positivo, pero porque yo como que nunca quise llevarle un trabajo para que usted lo viera [risas de algunas chicas]; yo me sentía cada vez más tranquila eh, mostrándole a mis compañeros: ¡Ve, corrígeme aquí tal cosa, qué pasó!, o o sea [de nuevo risas de los chicos], o sea para mí fue positivo en este sentido [...]. Sí, sentía más confianza, llegar a la clase y bueno esto no lo va ver el profesor así de primeras, sino que tengo tiempo, bueno me van a corregir, eh tengo tiempo para modificar lo que tengo que modificar, y al final, el profesor lo va a recibir, un trabajo mejorado.

Es necesario aclarar que durante el desarrollo de la práctica, el profesor distribuía al azar los trabajos, evitando precisamente que los estudiantes fueran revisados por sus amigos o personas más cercanas, buscando mayor objetividad y rigor, - aunque estos objetivos no se alcanzaran muchas veces-, también para que los estudiantes tuvieran presentes otros puntos de vista y opiniones sobre su trabajo y también para crear un ambiente de trabajo más cooperativo, para que hubiera mejor integración en el grupo.

\section{Desajustes en las concepciones y creencias sobre el papel del profesor durante la implementación de la práctica de revisión entre iguales}

Los estudiantes reconocen que la mediación del profesor es fundamental en la enseñanza de la revisión entre iguales, pero algunos se mostraron sorprendidos por el rol del docente frente a la práctica. El hecho de centrar el eje de la actividad en el aula en la revisión entre iguales, provocó que el profesor perdiera el protagonismo que suele tener el docente en el aula universitaria para desplazar el centro de atención hacia la actividad de los sujetos. Quizás los estudiantes no estaban preparados para asumir de manera tan rápida la responsabilidad de la revisión de sus propios trabajos y los de sus compañeros dentro del aula, situación a la que les costó trabajo adaptarse, habría que tener en cuenta el hecho de que llevar a cabo la práctica dentro del aula - cuando no se tienen experiencias previas de revisión personal y conjunta- puede producir una mayor carga cognitiva y de estrés emocional. Se podría combinar en posteriores trabajos con la actividad fuera del aula, en la que los estudiantes se llevaran el trabajo de su pareja de escritura y lo trajesen revisado en la sesión siguiente. Muy seguramente sea necesario ajustar, 
hacer una graduación, una entrega paulatina de la responsabilidad a los estudiantes.

La cesión de la responsabilidad a la que hemos hecho referencia en esta secuencia de aprendizaje también produjo desajustes en el mismo docente frente a su nuevo papel en el aula. Esto produjo algún desacomodamiento en las representaciones de algunos alumnos sobre el papel tradicional del docente. Su "ausencia" fue interpretada en un sentido paradojal; por ejemplo, Dora consideró que se trataba de un "ausentismo positivo":

Dora: Pues todos consideramos que la mediación del profesor era fundamental, pero digamos en su curso usted siempre evitaba precisamente eso, o sea como podía retirarse un rato y dejarnos ahí solos, y como que no lo viéramos para que no le llegáramos con todos los textos, eh eh, era como, o sea, como dejándonos muy solos hasta que nos acostumbráramos $[\ldots]$.

Esta situación produjo un desconcierto inicial a la que los estudiantes se fueron acomodando por la fuerza de la costumbre:

Andrea: Pues uno al principio no sabe cómo manejar la situación porque uno está es acostumbrado es que el profesor esté ahí pendiente, y ¡usted escriba y usted también!; uno al principio no sabe cómo va a manejar la situación, de cómo está al el profesor y tengo una pregunta y solamente le puedo preguntar al profesor, entonces eh, pero como decía Dora ahora, uno luego se va acostumbrando a que hay momentos en los que bueno el profesor está, bueno entonces ya está como más organizado, ya nos acostumbramos a llevar el proceso, pues con la ayuda del profesor, pero prácticamente lo hacíamos entre nosotros mismos.

Finalmente, uno de los informantes plantea que el profesor no estaba buscando una nota y que esto producía una mayor confianza para mostrarle los borradores.

Gustavo: El profesor no estaba buscando eh una nota, sino que estaba buscando otra cosa [...]; yo creí que era como todos los profesores, que estaba haciendo talleres buscando una nota o algo, entonces yo tenía ese miedo, entonces yo no le entregaba nunca los trabajos, mejor que lo critique otro que me dicen los errores, pero no me dan ninguna nota [...]; yo creí que era un estilo muy propio, era diferente; él no estaba buscando tanto una calificación sino como que [“¡usted desarrolle sus habilidades!”, interrumpe Andrea en un solapamiento de turno] ¡sí!, pues no tanto eso, sino que era como que, que cómo se está desenvolviendo el estudiante, como un proceso, porque usted nos daba unos estilos muy propios, usted se retiraba de la clase y nos dejaba, o nos daba un texto y nos poníamos a escribir sobre él o después con la biografía, entonces pues yo me di cuenta que al fin era un estilo muy diferente, entonces ahí, pues me tomé más confianza y pues ahí yo comencé pues a mostrar un poquito mis textos y pues a dialogar con usted sobre esto.

El significado que los estudiantes atribuyen a la transición entre colegio y universidad en relación con sus procesos de construcción de la escritura académica

Para los participantes en el grupo de discusión, existe un punto de inflexión marcado en el paso del bachillerato (educación media o secundaria) a la universidad. Lo que revelan sus discursos es que no tuvieron la oportunidad de estar abocados a prácticas de escritura académicas ni siquiera en los grados superiores del bachillerato y que estas eran prácticamente desconocidas para ellos.

No tenían conciencia, por ejemplo, sobre el problema de los géneros, específicamente del ensayo académico. Los saberes sobre los géneros y las prácticas fueron construidos ya en el ámbito de la educación superior.

Adela: En el colegio, en el colegio a mí nunca me pusieron a hacer un ensayo; yo vine a escuchar la palabra ensayo en la universidad, ¡seguro!

Gustavo: Uno creía que, como un resumen, era como la estructura de un resumen.

Jorge: Es que en el colegio, en el colegio a uno lo ponían a escribir ensayos y uno pensaba pensaba que simplemente era coger dos ideas y contrastarlas y iya!; pero entonces aquí uno ya se viene a dar cuenta cuando uno realiza un ensayo, así, bien largo que un curso de casi 6 meses, uno se 
da cuenta de que es bastante complejo porque son varias, son analizar todas las ideas; primero se tiene que hacer la macroestructura, definir cuál es la hipótesis, bien cuál es el tema, definir cómo va a ser uno de los párrafos a las proposiciones y cada párrafo trabajarlo y después como un ente aparte y al final tener todo combinado, pues es entonces bastante complejo realizar un ensayo.

Por otra parte, el ingreso a la universidad supone romper con un pasado en el que la rutina y la rigidez de las prácticas educativas incidieron negativamente en la construcción de saberes sobre la lectura y la escritura. Al contrario, la lectura ocupó también un lugar significativo en el comienzo del proceso de aculturación académica de los estudiantes.

Esteban: Bueno, pues para mí, lo más importante, lo que yo considero más importante del curso, eh, fue al amor por la lectura, que, a mí, no me. Yo llegué a la universidad y no me gustaba leer, por, yo en el colegio ni siquiera leía, porque ni siquiera leía; yo creo que ese es un punto primordial porque la lectura y la escritura están están, van de la mano, entonces crear eso, el amor por la lectura, pues incide demasiado en el en el proceso de escritura también.

Pedro: Yo estoy de acuerdo con lo que dice Esteban. Precisamente, el cambio de esa metodología psicorígida donde te ponen, te exigen, donde te ponen con algo que no estás a gusto, entonces pasamos a esa parte, el curso I [Composición en Español I], donde donde estas estas lecturas te motivan, las lecturas que son muy, son muy atractivas, te ponen, te ponen a pensar, te ponen como a reflexionar, todo eso te te; a mí me ponían; yo aprendí a escribir también acá en la universidad.

El significado que le dan a la experiencia de la enseñanza de la revisión entre iguales y de los procesos de composición de textos académicos en su formación como futuros docentes de lenguas

Este es un aspecto sobre el cual el docente insistió mucho a lo largo de la secuencia de aprendizaje, por lo que pudo haber condicionado de algún modo el pensamiento de los estudiantes. No obstante, explorar el significado que atribuyen a su futuro como docentes de lenguas es un aspecto importante porque los estudiantes pertenecen a un programa de formación docente. Interesa destacar que a los estudiantes les gustaría implementar, de algún modo, el modelo aplicado en la secuencia de aprendizaje, brindando mayor autonomía y responsabilidad a sus futuros estudiantes, confianza en sí mismos y en sus propios compañeros, así como la capacidad de regularse a sí mismos.

Dora: Crear un poquito más de autonomía en los estudiantes, o sea que dejen igual que nosotros, el que vayan empezando a pensar que no necesariamente tiene que estar el profesor ahí dándoles el visto bueno con todo, sino que ellos mismos creen sus formas de evaluación, y pues se den confianza.

Jorge: Y así como profesores lo que vamos a hacer tenemos la oportunidad de cambiar el enfoque pedagógico que se sigue llevando aquí todavía y en Latinoamérica, el tradicionalista, y poder cambiar hacia ese tipo de enfoque donde se le da confianza al estudiante en su propio trabajo, en la confianza del estudiante en sus compañeros también.

Moisés: Darse cuenta que cuando uno está estudiando o cualquier otra actividad en la vida, uno también tiene otras personas que están haciendo las mismas cosas que uno está haciendo y que, o sea estamos compartiendo como un, como unas mismas vivencias, y de los modos de ver dentro del aula es una manera como de de de tener puntos de vista de, digamos eso tiene que servir para para y para ubicar o para comunicar eso a las personas que va a estar en cargo de nosotros y en en un aula de clase y que puedan compartir sus vivencias con otros, con los compañeros de clase.

\section{Conclusiones}

El análisis del grupo de discusión permitió describir y analizar, desde la perspectiva del estudiante, las representaciones, opiniones y el grado de conciencia de los alumnos sobre la práctica de la revisión entre iguales y su incidencia en el proceso de construcción de la escritura académica que ingresaban por primera vez a la universidad a cursar estudios de Licenciatura en Lenguas Extranjeras. 
La práctica de revisión entre iguales produjo al inicio de la secuencia de aprendizaje resistencias y temores representados en la desconfianza sobre la competencia del estudiante para revisar el trabajo de su compañero, fundamentada, a su vez, en la creencia de la falta de criterios y experiencia para revisar el trabajo propio. Se trata de concepciones muy arraigadas en los estudiantes que participaron en la secuencia de aprendizaje. Este hecho plantea la necesidad de estructurar o mediar mejor la práctica para enfrentar estas resistencias, probando otras estrategias como solicitar a los estudiantes que se lleven el borrador a casa y lo traigan revisado, con una hoja o esquema de las principales observaciones $y$ correcciones para ser discutidas con el autor en la clase o clases siguientes. También se podría desarrollar la práctica de revisión entre iguales dentro del aula sin rejillas de evaluación previas, es decir, dejar a los estudiantes que revisen con mayor libertad y determinar en qué aspectos de los textos se concentran y cómo lo hacen, qué actitudes y valoraciones tienen, etc., así como combinar este tipo de prácticas a lo largo de la secuencia de aprendizaje.

Hay que tener en cuenta que la revisión entre iguales se llevó a cabo in situ, en el aula, y esto puede suponer una mayor carga cognitiva y emocional. Habitualmente los docentes de escritura en la universidad piden a sus estudiantes que escriban sus trabajos fuera del aula y suponen que estos revisan como escritores expertos cuando no lo son.

Sin embargo, en el discurso de los participantes en la entrevista, se evidenció que, a pesar de que las resistencias se mantuvieron durante la secuencia, estas reticencias cedieron un poco y dieron lugar a situaciones paradójicas: la de alumnos que preferían ser revisados por un compañero, antes que por el docente, porque esto les permitía hacer varias correcciones antes de entregarle una versión más elaborada y la de aquellos que preferían buscar la revisión del profesor, así fuese en una segunda instancia después de ser revisados por un par porque consideraban que las correcciones de sus compañeros eran demasiado superficiales (puntuación, ortografía, algunas repeticiones, etc.).
Con todo, los estudiantes valoran positivamente la práctica de revisión entre iguales porque les ayudó a tener más confianza en sí mismos y a tomar mayor conciencia sobre el proceso de composición escrita de textos académicos, sobre la necesidad de revisar los textos propios de manera más seria y exhaustiva, de releer los textos académicos que escriben, de la audiencia para la que escriben, de la estructura de los textos académicos y de la construcción discursiva que soporta el texto en términos de la coherencia y la cohesión. Ahora bien, dos alumnos mencionaron la necesidad de continuar este tipo de prácticas, porque un solo curso no es suficiente y, por otra parte, necesitamos más documentación y otros ciclos de estudio que permitan indagar si realmente los estudiantes han interiorizado el subproceso de la revisión, si revisan más y mejor y si solicitan a otros las revisión de sus textos académicos o la ofrecen a otros, después de haber finalizado este curso.

Hubo posturas antitéticas en la valoración del rol del docente: el profesor creyó haber intervenido mucho en diversas instancias de la secuencia de aprendizaje, especialmente durante las sesiones de revisión en pareja, mientras que los estudiantes argumentaron que había desaparecido casi de la escena y experimentaron muchas dificultades para asumir esta cesión de responsabilidad en el aprendizaje de la escritura académica. Este es un aspecto para revisar en próximas secuencias de aprendizaje y otros ciclos de estudio.

En conjunto, hubo varias críticas a la educación recibida durante la primaria y la secundaria porque no preparó a los alumnos para la escritura de textos académicos en la universidad, crítica que supuso discutir los modelos pedagógicos dominantes en estos niveles de escolaridad donde el profesor detenta el poder que le confiere su estatus y su saber y el alumno no tiene ningún grado de autonomía.

Para la mayoría de los estudiantes su ingreso a la universidad, y de manera particular la secuencia de aprendizaje desarrollada en el curso de composición escrita objeto de este estudio, representó un punto de inflexión en su historia como lectores y escritores. Muchos defendieron la idea que fue en 
Universidad Pedagógica Nacional

Facultad de Humanidades

la universidad donde aprendieron a escribir, donde por primera vez en sus vidas tomaron conciencia del proceso de escritura, de los tipos de textos, de las estructuras textuales, de la coherencia y cohesión que deben tener los textos académicos, entre otros conocimientos.

El papel de la lectura en el desarrollo de la secuencia de aprendizaje también fue destacado por varios alumnos. Lo consideraron decisivo; para algunos fue un encuentro revelador porque no leían en el colegio, porque los textos que se leyeron en el aula (reseñas, ensayos y artículos académicos) resultaron motivadores, placenteros y porque, según sus palabras, invitaban a reflexionar. Además, porque estos constituyeron una buena provocación para aprender a escribir de forma académica, con placer también, en un ambiente que los alumnos calificaron como cálido y donde daba gusto estar, donde daba gusto aprender. Sin embargo, el tema de la lectura no fue abordado como objeto específico en el grupo de discusión ni en la secuencia didáctica.

Por último, cabe comentar que el dispositivo de revisión entre iguales es más complejo de lo que parece y que requiere un andamiaje especial, reajustes permanentes, pero que puede contribuir a desarrollar prácticas de aprendizaje de la escritura académica más dialógicas y democráticas en la universidad, generalmente monológicas y dominantes, y que considerar la perspectiva del estudiante en este proceso desde las literacidades académicas puede llegar a ser una línea muy fértil de trabajo.

\section{Referencias bibliográficas}

Boote, R. y Beile, P. (2005). Scholars before researchers: On the centrality of the dissertation literature review in research preparation. Educational Researcher, 34(6), 3-15.

Brown, G. y Yule, G. (1983). Discourse Analysis. Cambridge: Cambridge University Press [Versión española: Análisis del discurso. Madrid, Editorial Visor (1993)].

Camps, A. (1994). L'ensenyament de la composició escrita. Barcelona: Barcanova.
Carlino, P. (Octubre 8-9, 2004). Diario de tesis y revisión entre pares: análisis de un ciclo de investigaciónacción en talleres de tesis de posgrado. Ponencia presentada en las I Jornadas Latinoamericanas Investigación y Práctica en Psicología Educacional. Universidad de Buenos Aires, Buenos Aires.

Carlino, P. (2005). Escribir, leer y aprender en la universidad. Buenos Aires: Fondo de Cultura Económica.

Carlino, P. (2008). Revisión entre pares en la formación de posgrado. Lectura y vida, 2, 20-31.

Cassany, D. (1993). Reparar la escritura. Barcelona: Editorial Graó.

Cassany, D. (1999). Construir la escritura. Barcelona: Paidós.

Cassany, D. y Morales, O. (2009). Leer y escribir géneros científicos. En D. Cassany (comp.), Para ser letrados. Voces y miradas sobre la lectura (pp. 109-123). Barcelona: Paidós.

Castelló, M. (2009). Escribir trabajos de investigación con alumnos de grado. Textos, 50, 21-29.

Castelló, M. (2008). Usos estratégicos de la lengua en la universidad. Tácticas de regulación de la escritura en estudiantes de doctorado. En A. Camps y M. Milian (coords.). Miradas y voces. Investigación sobre la educación lingüística y literaria en entornos plurilingües (pp. 75-90). Barcelona: Graó.

Castelló, M., González, D., e Iñesta, A. (2008). Socially shared regulation in writing. 11th International Conference of SIG Writing. Lundt. <http:sig-writing. publication-archive.com/public?fn=enterEtreposit ory $=1$ Earticle $=410$ (Consulta: 22 de octubre 2008).

Dysthe, O., Samara, A. y Westheim, K. (2006). Multivoiced supervisión of Master's students of alternative supervisión practices in higher education. Studies in Higher Education, 31(3), 299-318.

Duranti, A (1997). Antropología lingüística. Madrid: Cambridge [Edición original: Linguistic antropoligy. Cambridge: Cambridge University Press].

Flower, L. (1994). The construction of negotiated meaning. A social cognitive theory of writing. Carbondale and Edwardsville: Editorial Southern Illinois University Press.

Gee, J. P. (1996). Social linguistics and literacies. Ideology in Discourses. London and New York: Routledge. [Versión española: La ideología en los discursos. Madrid: Editorial Morata, 2005]. 
Revisión entre iguales y escritura académica en la universidad: la perspectiva del estudiante Alfonso Vargas Franco

Ivanic, R. (1998). Writing and identity. The discoursal construction of identity in academic writing. AmsterdamPhiladelphia: John Benjamins Publishing Company.

Lillis, T. (1997). News voices in academia? The regulative nature of academic writing conventions. Language and Education, 11(3), 182-99.

Lillis, T. (2001). Student writing. Access, regulation, desire. London and New York: Routledge.

Lillis, T. (2003). Student writing as Academic Literacies: Drawing on Bakthin to move from critique to design. Language and Education, 17(3), 192-207.

Payrató, L. (2003). Pragmática, discurs i llengua oral. Barcelona: UOC.

Scollon, R. y Scollon, S. W. (1981). Narrative, literacy, and face in interethnic community. Oxford: Blackwell.

Starke-Meyerring, D. (2010). Between peer review and peer production: genre, wikis, and the politics of digital code in writing. En Ch. Bazerman, et al., Traditions of writing research (pp. 339-350). New York: Routledge.

Tusón, A. (1997). Análisis de la conversación. Barcelona: Ariel.

Vargas Franco, A. (2005). Escribir en la universidad: reflexiones sobre el proceso de composición escrita de textos académicos. Lenguaje, 33, 97-125 [Cali: Programa Editorial Universidad del Valle].

Vargas Franco, A. (2008). Escribir en la universidad (Reflexiones y estrategias sobre el proceso de composición escrita de textos académicos) [2a ed.]. Cali: Programa editorial de la Universidad del Valle.

Vargas Franco, A. (2010). Concepciones y representaciones sobre la escritura en la universidad: el caso de la revisión entre pares. En C. M. Maya Franco y A. A. Puerta Molina (ed.), Cultura escrita en la universidad (cap. 2, pp. 25-37). Medellín: Universidad de Medellín.

Vargas Franco, A. (2013). Revisión entre iguales, escritura académica e identidad en la formación docente en una universidad colombiana. Tesis doctoral no publicada. Barcelona: Universidad Pompeu Fabra. www.tdx.cat/bitstream/handle/10803/125115/tavf. pdf? sequence=1 Consulta: 13/02/2014.

Wells, G. (2001). Indagación dialógica. Barcelona: Paidós.

Zavala, V. (2009). ¿Quién está diciendo eso?: literacidad académica, identidad y poder en la educación superior. En J. Kalman y B. Street (eds.), Lectura, escritura y matemáticas como prácticas sociales. Diálogos con América Latina (pp. 348-363). México: Siglo XXI.

Zavala, V. (2011). La escritura académica y la agencia de los sujetos. Cuadernos comillas. Revista Internacional del Aprendizaje del Español. Fundación Comillas. El Español escrito en contextos contemporáneos. Monográfico 1, 52-66.

Zavala, V. y Córdova, G. (2010). Decir y callar. Lenguaje, equidad y poder en la universidad peruana. Lima: Pontificia Universidad Católica del Perú. 